\title{
Research on the Development of Internet of Vehicles Technology
}

\author{
Ying Pan \\ College of Physics and Electronics Information Engineering, Qinghai Nationalities University, Xining, China \\ Email address: \\ 15709784650@139.com

\section{To cite this article:} \\ Ying Pan. Research on the Development of Internet of Vehicles Technology. Internet of Things and Cloud Computing. \\ Vol. 7, No. 1, 2019, pp. 12-18. doi: 10.11648/j.iotcc.20190701.12
}

Received: December 24, 2018; Accepted: January 15, 2019; Published: February 4, 2019

\begin{abstract}
Internet of vehicles (IOV) is a large interactive network composed of vehicles' position, speed, route and other information. Through global positioning system (GPS), radio frequency identification (RFID), sensor, camera image processing and other devices, the vehicle can complete its own environment and state information collection; Through Internet technology, all vehicles can transfer all kinds of information to the central processing unit (CPU). Through computer technology, this mass of vehicle information can be analyzed and processed to calculate the best route for different vehicles, timely report the road condition and so forth. The development of IOV will have a comprehensive driving effect on social construction in many aspects, such as intelligent traffic management, energy conservation, emission reduction and safe driving. In general, mature industrial base, huge consumer market and important strategic significance make the IOV become the focus of large-scale development and application of the IOV in the industry in the world, which has won strong attention from all parties. There is no strict definition for the so-called IOV. To put it simply, it is to realize the coordinated interaction of people, vehicles, roads and the environment through wireless communication and other means by taking cars as nodes in the information network, so as to realize intelligent transportation. However, since its birth, the IOV has always been faced with the lack of a unified management situation. In this paper, we studied the main technology involved in the IOV, the existing problems and so on.
\end{abstract}

Keywords: Internet of Vehicles (IOV), Global Positioning System (GPS), Radio Frequency Identification (RFID), Sensor

\section{Introduction}

The term IOV is not a foreign product, there is no strict corresponding English word abroad. Foreign research on IOV mainly focuses on intellectual transportation system (ITS) and telematics system, which can be regarded as the two focuses of IOV. ITS is a general term for the integrated application of communication, control and information processing technology in the transportation system. Telematics is a combination of telecommunications and informatics, namely, remote information service. It can be understood as an on-board system service platform that provides entertainment, rescue, navigation and other conveniences for automobile users through wireless communication technology, satellite navigation system, network communication technology and so on [1-4].

From specific definition, IOV refers to the load on the vehicle electronic sensing devices, through the wireless identification such as information technology, information network platform for all attribute information of the vehicle and static or dynamic information extraction and effective utilization, and according to the different functional requirements of vehicles running state comprehensive management and effective service. Its ultimate purpose is to use information technology, wireless communication technology and other information on the vehicle and outside the analysis and use. To realize the omni-directional communication between vehicles, roads and people. The IOV organically applies advanced sensing technology, communication technology, Internet technology, cloud computing technology, intelligent technology and control technology into the vehicle and traffic road management system to build a real-time, accurate and efficient comprehensive traffic and transportation management and control system. Through the formation of information interaction between vehicles and drivers, road facilities, 
service providers, Internet and other information, the dynamic interaction between vehicles and roads, vehicles and vehicles, vehicles and people is realized. On the overall level, more intelligent and safe driving can be achieved and professional information application services can be enjoyed [5-12].

IOV is a subdivision application of the IoT in the automotive field. Its essence is the integration of the IoT and the mobile Internet. It is the future development technology of information communication, environmental protection, energy conservation and security in the field of intelligent transportation. It fundamentally changes the state in which vehicles have existed as information islands for a long time, making each vehicle a node in the IoT, thus realizing a comprehensive perception of roads and traffic, with largescale and large-capacity between multiple systems. The interaction of data enables full control of each vehicle, full time and space control for each road, and more additional services for the driver, which has great application potential [13-15].

\section{Framework of IOV}

The IOV system mainly includes three parts: vehicle terminal, cloud computing processing platform and data analysis platform. By vehicle and road, vehicle and vehicle, vehicle and people, and the connectivity to realize information sharing, information collection vehicles, roads and environment, and on the network information platform for multi-source collection and information processing, calculation, share and publish, with vehicles as the carrier to carry out information service, can effectively solve the road, the vehicle, the comprehensive coordination. Using various technical means to connect vehicles with roads, vehicles with vehicles, and vehicles with people, which is the existence form of IOV technology. Various products and services developed on this basis are the core of IOV. The IOV system composition is shown in figure 1 .

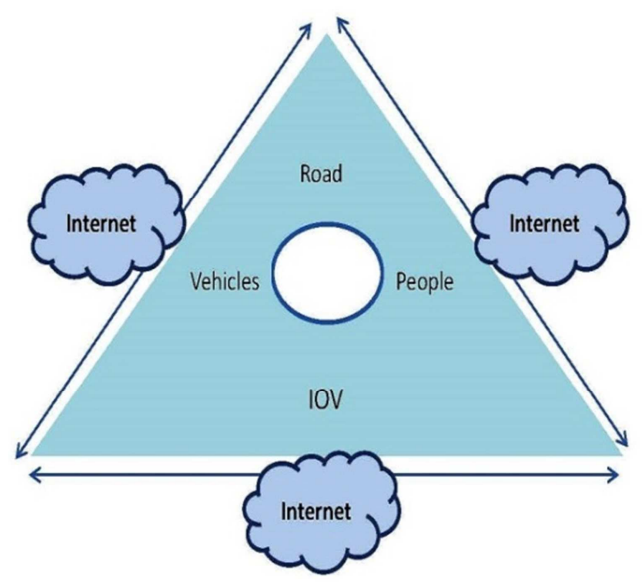

Figure 1. IOV system composition.

In essence, the bus network is the IoT in an application of the auto industry, its architecture and the IoT typical architecture, mainly completed by providing business application layer, data transmission network layer, and provide business application layer. Of course, in order to achieve the massive amounts of business application, also needs the support of cloud computing as an information service. The core architecture is shown in figure 2 .

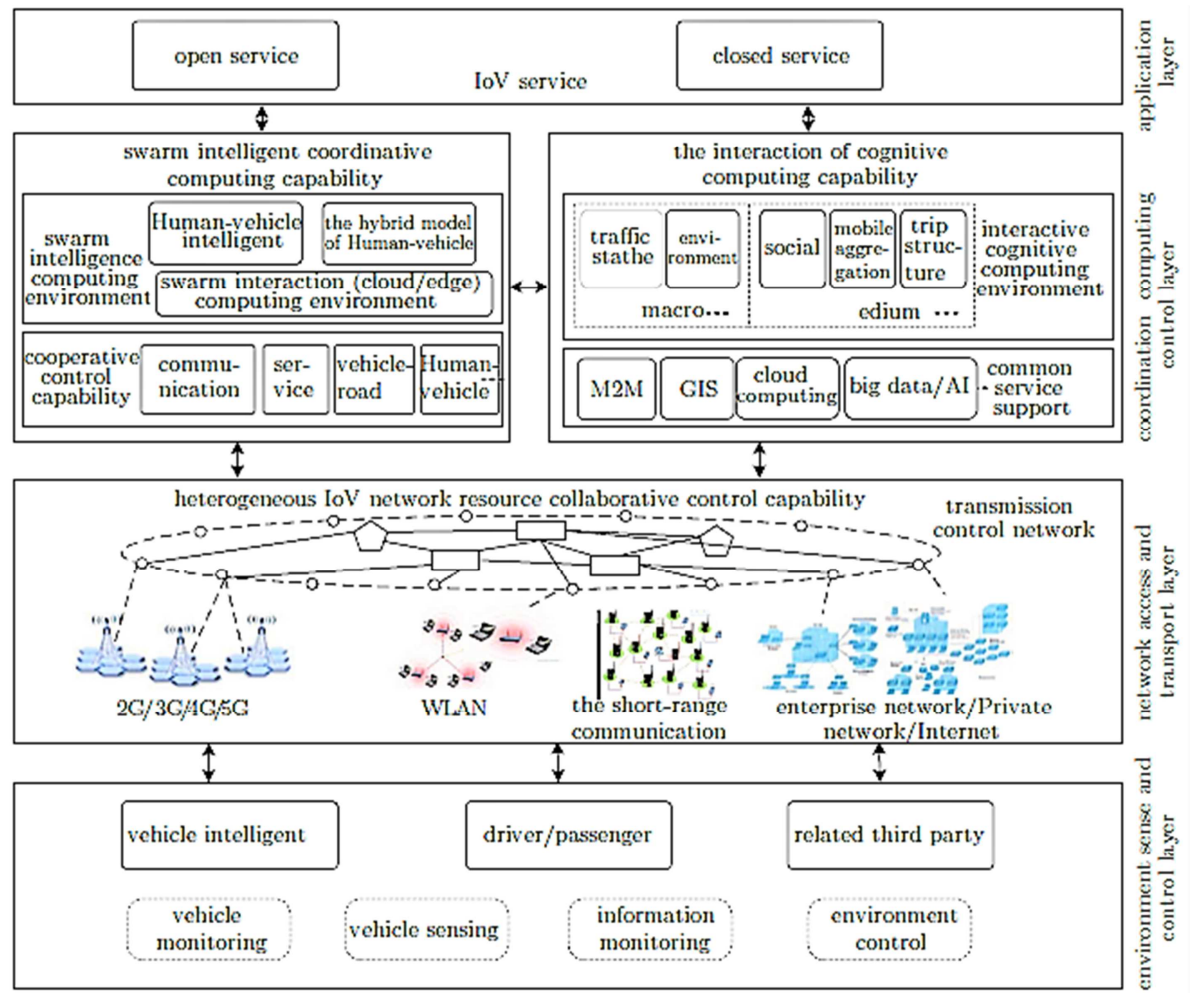

Figure 2. IOV architecture. 
The lowest layer of the architecture of IOV is the basic perception layer, which is the nerve endings of the IOV. It is usually composed of all kinds of sensory terminals distributed in vehicles, roads and the surrounding environment. In its current form, it mainly consists of smart phones, on-board smart terminals, monitoring equipment, road or vehicle sensors and other types of equipment. This layer realizes information perception and information collection of vehicle-to-vehicle, vehicle-to-road with the help of sensing technology, satellite positioning technology and intelligent and control technology. This layer is the source of data information of IOV.

The second layer is the network layer, namely the communication layer, which is mainly used to transmit all kinds of sensory information collected in the first layer. At present, all kinds of wired and wireless, long-distance and short-distance, narrow-band and broad-band communication systems are used as the channels for information transmission.

The third layer is the application service layer, which usually builds the business cloud with the help of cloud computing and distributed data service center. Through the calculation, analysis and comprehensive processing of the data collected from the perception layer, after processing and integration, it provides users with various information services and applications such as traffic conditions, tourism navigation, video and audio entertainment and so on. Business applications get through the three links of road, vehicle and people, and constitute an organic combination of the three. Each part is the provider of information in the IOV, and finally becomes the user of information. Therefore, the application layer is the core layer of the IOV.

\section{Key Technologies of IOV}

The IOV needs to integrate the existing electronic information technology, so that each vehicle becomes an intelligent information terminal. With the help of wireless communication means, build a network covering all the vehicles that can reach the place, and achieve $7 * 24$ hours online information interaction. Its ultimate goal is to achieve optimal intelligent traffic management, to achieve the highest efficiency of the use of road facilities, more secure and comfortable driving experience and more energy-saving and environmentally friendly road travel. It mainly involves sensing technology, intelligent terminal, speech recognition, cloud computing, wireless communication, mobile Internet and other key technologies.

\subsection{Multi-information Fusion Technology of Multi-sensors}

The IOV is the network among vehicles, roads and people, and its focus is the sensor network between vehicles and roads. The sensor network of vehicles can be divided into invehicle and off-vehicle sensor networks. The in-vehicle sensor network is responsible for providing vehicle attributes and static and dynamic vehicle status information to people.
The external sensor network is used to perceive the external environment of the vehicle. The sensor network of the road is used to perceive and transmit the road information. Whether inside or outside the vehicle, or the road sensor network, all play the role of environmental perception, for the IOV to obtain unique information. Integration of these information, that is, integration of sensor network information is an important characteristic of the IOV technology development content. By using computer technology to automatically analyze, synthesize, reasonably combine and dominate these sensors and observation information under certain criteria, it can provide comprehensive help for the development of various applications of IOV.

Perception technology provides data sources for IOV. IOV sensing technology mainly includes: common automotive sensors, data bus, automatic control and fuzzy control technology, video image recognition, radar speed measurement and laser speed measurement, geomagnetic sensing detection, laser ranging, ultrasonic ranging and so on. In recent years, micro-electromechanical systems (MEMS) technology developed from semiconductor integrated circuit technology is becoming increasingly mature. This technique can be used to make micro sensors to detect mechanical, magnetic, thermal, chemical and biomass. As MEMS microsensor has advantages in reducing the cost of automotive electronic system and improving its performance, the miniaturized, multi-functional, integrated and intelligent sensors based on MEMS technology will gradually replace the traditional sensors and become the mainstream of automotive sensors.

The implementation of IOV requires a large number of data sources. The traditional method is to lay induction coil on the road surface, set up ultrasonic sensor and image sensor to detect traffic flow and speed. At present, a new data acquisition method called floating vehicle information acquisition can use the vehicle as a mobile sensor to collect traffic data. At the technology of floating vehicle data collection, there are two key technologies, one is by various sensors over the body, including velocity, acceleration, gyro, GPS, tire pressure, as well as with other sensors to collect data in the field of auto safety and performance, using the advanced multi-sensor integration and data fusion technology, will be collected raw data into useful data transmission; Second, the body network construction. At present, the integration degree of automotive electronics is very high. How to manage the vehicle data and provide various raw data for the IOV without affecting the safety and performance of vehicles is also a difficult problem to be solved in the future.

\subsection{Open Intelligent Vehicle Terminal System Platform}

At present, many on-board navigation and entertainment terminals are not suitable for the development of IOV. The core reason is that the non-open terminal system platform is not smart enough. Based on the terminal system platform that is not open and not smart enough, automobile manufacturers 
can only provide services for their own brand of vehicle users. The service standards are not unified, and the quality of service varies greatly, which will make it difficult to create a benign network ecosystem. At present, the user terminals of the IOV include IOS system and Android system, etc. The terminal system platform of the IOV must be able to carry Android and iPhone platforms, such as iPhone, iPad, Android phone, Android navigator, Android tablet computer, etc. Only open system platforms can better serve users. According to the current situation, Google Android will also become the mainstream operating system of the IOV terminal system, and those closed operating systems may develop well in the early stage, but will eventually be restricted by open problems.

\subsection{Intelligent Speech Recognition Technology}

Due to the particularity of driving environment, peoplecomputer interaction in the era of IOV cannot use mouse, keyboard or touch screen, and voice interaction is safe and convenient, which naturally becomes the best way of peoplecomputer interaction. Sending control instructions through voice will become the booster for the development of IOV. The intelligent speech recognition technology is shown in figure 3.

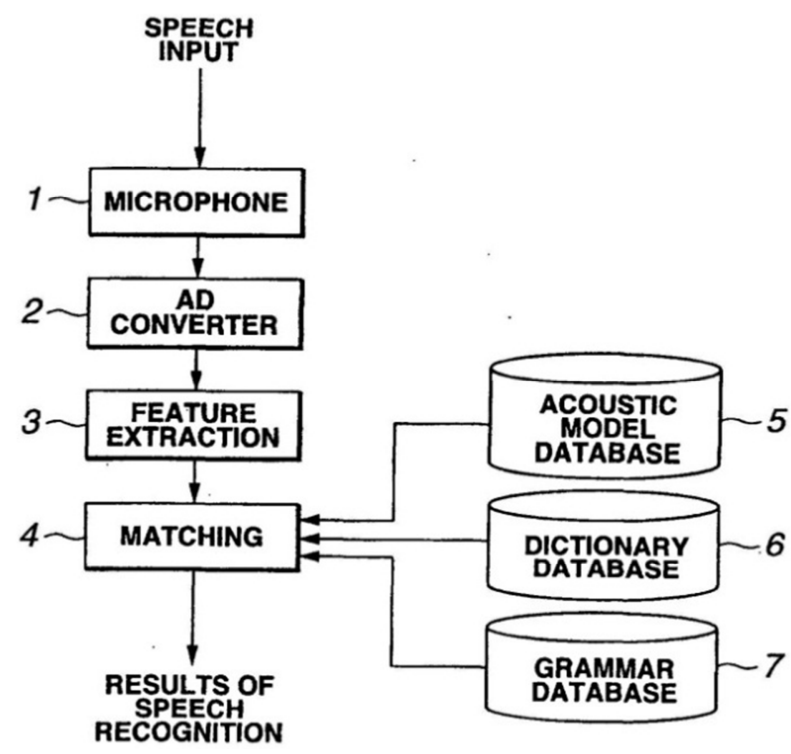

Figure 3. Intelligent speech recognition technology.

Intelligent speech recognition technology, which enables drivers to give orders by voice and receive services provided by the IOV with their ears, will be more suitable for the application experience of vehicles in this fast moving space. Mature speech recognition technology relies on the powerful corpus and computing power of the network, but neither the storage capacity nor the computing power of the on-board terminal can solve the problem of speech recognition technology of non-fixed commands. Therefore, it is necessary to adopt the cloud recognition technology based on the server-side technology to collect and calculate a large number of voice recognition data. Relying on the network computing technology, it is necessary to build a unique car voice network voice platform engine based on the mobile Internet environment to realize the recognition of multiple languages and even dialects.

\subsection{Cloud Computing}

Massive connections and massive data are bound to be generated in IOV applications. Cloud computing will be used in IOV to analyze and calculate road conditions, propose large-scale vehicle path planning, and calculate intelligent traffic scheduling, etc. Cloud computing, on the one hand, enables rapid business deployment and provides systematic telematics services for industry users in a short period of time. On the other hand, the platform has powerful computing power, latest real-time data and extensive service support, which can play a strong supporting role for the service. Traditional navigation is based on local data and is only a static road, while cloud navigation based on cloud computing can achieve real-time intelligent navigation. The cloud platform will adjust the planning in real time according to the needs of users and take into account the actual road conditions, emergencies and other factors to ensure that users always know the most practical and convenient route to arrive. The cloud computing is shown in figure 4.

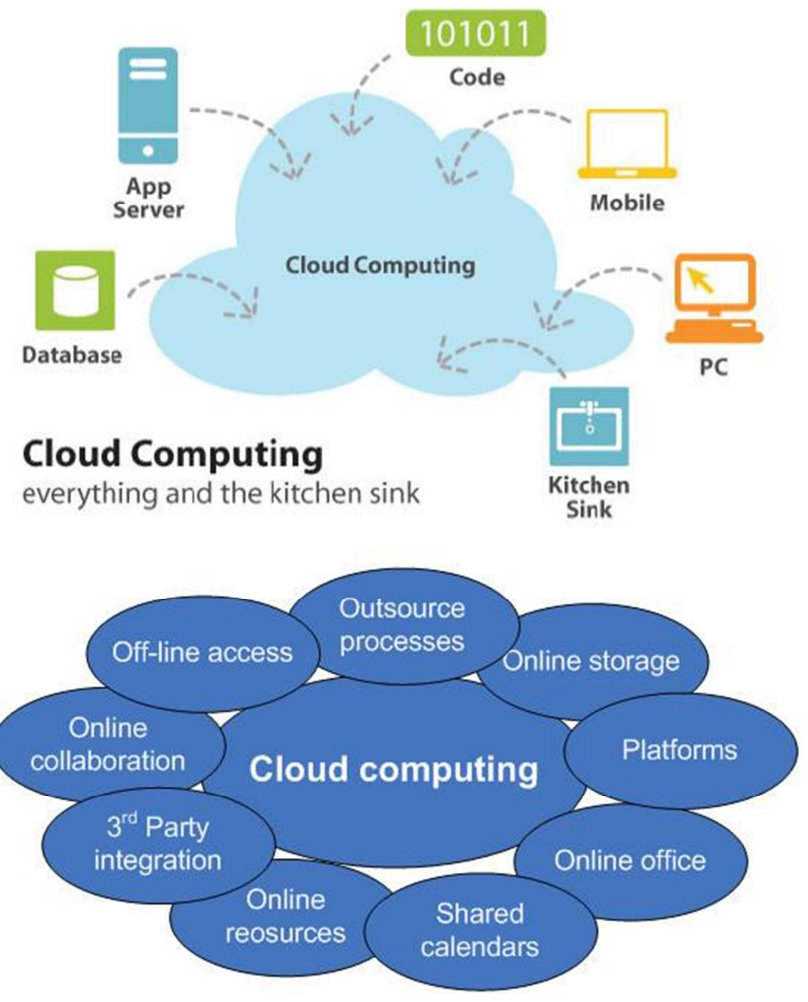

Figure 4. Cloud computing.

By integrating more information and resources through the service computing technology and platform of cloud collaboration, service innovation and value-added services can be realized. Through the integration and innovation of services, vehicle-mounted terminals can obtain more appropriate and valuable services, such as the integration of 
call center services and auto insurance business, remote diagnosis and on-site service reservation integration, location service and merchant service integration, etc.

In the field of intelligent transportation, there are currently serious information islands. Difficulty in information sharing between systems and delay in information transmission will restrict the accessibility and accuracy of information transmission in the field of intelligent transportation, and affect the efficiency of decision support and management scheduling. The special solutions of cloud computing technology, such as super computing power, dynamic resource scheduling, on-demand service provision and mass information integration management mechanism, will become important means to solve this problem.

\subsection{Navigation and Location-Based Services}

The GPS developed by the United States and Galileo positioning system of the European Union, Glonass system of Russia and Big Dipper positioning system of China have become the four core suppliers of global satellite navigation system recognized by the United Nations satellite navigation committee. Navigation and positioning technology greatly supports the application of IOV.

The navigation system of automobile mainly consists of two parts: navigation host and navigation display terminal. The antennas receive data from satellites orbiting the earth to determine the vehicle's current position. The position coordinates determined by the navigation host through satellite signals are matched with the electronic map data so as to determine the exact position of the vehicle in the electronic map. On this basis, it can realize navigation, route recommendation, information query and other functions. Drivers only need to watch the screen on the monitor, listen to the voice prompt, the remote control in the hand can achieve the above functions, so as to drive easily.

The application of automobile navigation system cannot be separated from the data provided by electronic map, which is of great significance for supporting various upper applications of IOV. At present, electronic map has been widely used for navigation, traffic sign prompt, real-time dynamic path planning and various location services.

Location-based services of the IOV can be divided into two categories: traditional services and new services. Traditional services focus on the integration of service industry chain. The services provided are mainly navigation services, including hot spot location information search (such as restaurants, entertainment, gas stations, etc.), information push, weather warning, automobile service information, etc., which are usually static or one-way information. New services, on the basis of the application of combined with huge amounts of mobile Internet users, through the vehicle networking community forming many more interactive applications, such as location information sharing, custom vehicle traffic information generated, exchange of experience, based on the location of the discount information, etc. It can according to user's requirements and technology development, constantly to the surrounding stretch, let the inherent service gradually have self innovation vitality, for the user's work, life and entertainment to bring more convenience.

\subsection{Wireless Communication Technique}

The IOV mainly adopts two wireless communication technologies, namely, short-range wireless communication technology and mobile wireless communication technology. The former is mainly based on RFID, Zigbee and Wifi. Mobile wireless communication mainly refers to General Packet Radio Service (GPRS), the 3rd generation telecommunication $(3 \mathrm{G})$, the fourth generation of mobile phone mobile communication technology standards $(4 \mathrm{G})$ and future the 5 th generation wireless systems (5G) and other mobile communication technologies. This two kinds of communication technology is not connected to the unique technology, so the technology development main emphasis is the communication technology in the field of vehicle networking applications, including highways and parking lot automatically capture to expend, short distance wireless communication applications such as wireless Internet, voice over Internet phone (VoIP) applications (such as cyclists, team leading, etc.), video monitoring and mobile communication technology such as scheduling application. The wireless communication technique is shown in figure 5.

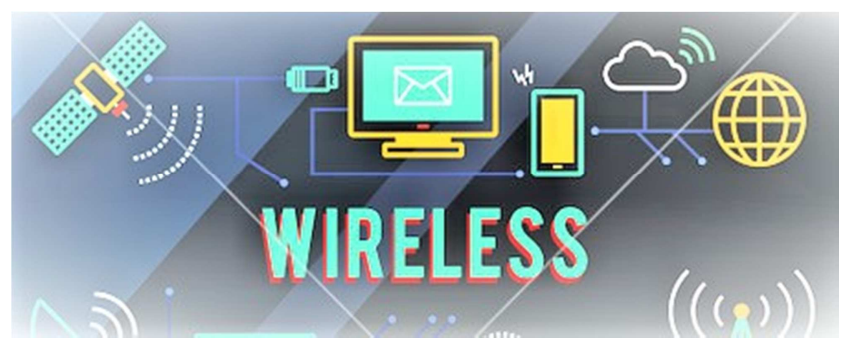

Figure 5. Wireless communication technique.

The main advantage of RFID applied to the IOV is that the technology can identify multiple objects moving at high speed and can easily realize data transmission between nodes in the IOV. In the IOV, active RFID technology is commonly used in the working frequency range of $5.8 \mathrm{GHz}, 2.4 \mathrm{GHz}$, $433 \mathrm{MHz}$, etc. Active RFID 5.8G ETC technology has been applied in the highway toll collection system. Passive RFID technology works in the frequency range of $840 \sim 845 \mathrm{MHz}$, 902 928MHz, which can be used for vehicle management. Zigbee technology based on IEEE802.15.4 standard is an emerging short-range wireless communication technology with low complexity, low power consumption and low cost. In some regional vehicle management, Zigbee technology is often used to track and locate vehicles and realize the intercommunication between vehicles.

Wireless communication technology is the key of IOV technology, which directly determines the real-time and effective information transmission. At present, there are various wireless communication technologies coexisting in the IOV, including wireless local area network (WLAN) (such as IEEE802.11a/g/n/p protocol), WiMax (IEEE802.16a /e), ultra-wide bandwidth (UWB) (IEEE802.15.3a), 2G/3G 
cellular communication, long term evolution (LTE), satellite communication and other networks. Different networks have different communication modes and characteristics, which are suitable for different scenarios. In order to achieve the purpose of information sharing, a lot of information in the IOV needs to be transmitted in different networks. In addition, as a mobile unit, the vehicle will undergo horizontal switching and vertical switching during the moving process, which also requires mobility management.

\subsection{Mobile Internet Technology}

When a variety of applications on smart phones are overwhelming, users are no longer satisfied with the basic navigation function on the vehicle system, but need products that support the mobile Internet just like smart phones. On vehicle network terminal, navigation is one of the many applications, there are many other applications based on user's location, such as drives, vehicle location tracking, sudden failure/accident report and so on, also can be in accordance with the requirements, micro letter, chat, music, games, such as application, meet the demand of the user and all applications in the field of auto life. Compared with the existing Internet and mobile Internet, IOV has two key features: one is related to vehicles and roads; the other is that location information is a key element. Therefore, it is more necessary to develop mobile Internet applications with IOV characteristics around these two key features.

At the same time, IOV, like other network systems, also faces many network security risks. Due to the complexity and diversity of the system, no specific security technology can completely solve all the security problems of the system. In addition, the IOV information security and personal privacy issues have been more and more attention. In the IOV environment, how to ensure the security and privacy of information, avoid virus attacks and malicious damage, prevent the loss of personal information, business information and property or theft by others, will be a major challenge to break through in the development of IOV. On the one hand, it requires continuous improvement in technology; on the other hand, it requires the formulation and improvement of relevant laws and regulations on the IOV, so as to provide a solid legal guarantee for the promotion and application of the IOV.

\subsection{IOV Proprietary Protocol LTE-V}

As the key research direction of pre-5G, LTE-V is a proprietary agreement for IOV. It was proposed by Chinese communication enterprises at the end of 2013. Huawei and Datang are the main standardisers of LTE-V.

LTE-V is aimed at the application scenarios of IOV, realizing the interconnection and data transmission between vehicles and vehicles $(\mathrm{V} 2 \mathrm{~V})$, vehicles and roadside facilities (V2I), vehicles and people (V2P), and vehicles and network (V2N), which are collectively referred to as V2X. The core of LTE-V is the interconnection of $\mathrm{V} 2 \mathrm{~V}$. In response to the vehicle active safety, efficiency, in-vehicle entertainment scene more business requirements, LTE-V combination widearea/hot cellular access technology (LTE-V-Cell) and shortrange distributed through technology (LTE-V-Direct), the former is based on the extension of the existing cellular technology, mainly for the traditional vehicle networking business, the latter is the introduction of LTE D2D (Device-toDevice), realize the V2V, V2I Direct communication, carrying vehicle active safety business, mainly to meet the requirements of the terminal security and low latency, high reliability.

LTE-V standard technical scheme has two ways:

Method 1: the control surface and user surface are forwarded by the operator network, and all V2X services can be provided.

Method 2: the user interface is realized through direct link, providing public $\mathrm{V} 2 \mathrm{X}$ services in addition to $\mathrm{V} 2 \mathrm{~N}$.

\section{Conclusion}

To sum up, in order to achieve the goal of intelligent transportation, IOV needs to rely on perception technology, satellite positioning technology, new generation communication technology, Internet technology, cloud computing technology, intelligence and control technology and other key technologies. Summarized in the following table:

Table 1. Key technologies of IOV.

\begin{tabular}{|c|c|c|}
\hline Category of technology & Functional description & Available technology \\
\hline Sensing technology & $\begin{array}{l}\text { Used for vehicle condition and body system perception, road } \\
\text { perception, vehicle and vehicle and road perception }\end{array}$ & Integrated sensor, RFID technology, diagnosis system \\
\hline $\begin{array}{l}\text { Satellite positioning } \\
\text { technology }\end{array}$ & Vehicle location, real-time navigation & GPS, Big Dipper \\
\hline $\begin{array}{l}\text { Wireless communication } \\
\text { technique }\end{array}$ & Safe, fast and efficient information exchange & $\begin{array}{l}2 \mathrm{G} / 3 \mathrm{G} / 4 \mathrm{G} / 5 \mathrm{G} \text { and other mobile communication technologies, } \\
\text { satellite communication technology, LTE-V technology, Wifi } \\
\text { technology, bluetooth } \mathrm{t} \text {, ZigBee }\end{array}$ \\
\hline $\begin{array}{l}\text { Cloud computing } \\
\text { technology }\end{array}$ & Data storage, processing, operation and mining, etc. & $\begin{array}{l}\text { Distributed server technology, road condition analysis and } \\
\text { calculation and path planning, intelligent traffic scheduling } \\
\text { calculation, remote analysis and diagnosis }\end{array}$ \\
\hline $\begin{array}{l}\text { Intelligent and control } \\
\text { technology }\end{array}$ & $\begin{array}{l}\text { Smart phones or on-board smart terminals have the function } \\
\text { of information collection and processing, and can actively } \\
\text { judge the vehicle body status, driver status and perceive the } \\
\text { external environment }\end{array}$ & $\begin{array}{l}\text { Audio and video intelligent analysis and identification } \\
\text { technology, intelligent control technology, automotive } \\
\text { electronic control and bus technology }\end{array}$ \\
\hline
\end{tabular}


In order to truly deepen people's life and develop rapidly, $\mathrm{IOV}$ in the future also needs to focus on solving a series of technical problems such as information collection and sharing, security, privacy protection and so on. In the future, the development focus of IOV should not be limited to "surfing the Internet", but should focus on connection, that is, communication. Only after the establishment of all-round communication between people and vehicles, between vehicles and vehicles, as well as between vehicles and roads, can information be transmitted and exchanged in a timely manner without hindrance can IOV play the most important role.

In order to better plan the construction and development of intelligent IOV, it is necessary for government departments to play a strong organizational and coordination function, formulate relevant policies and laws, support and guide the establishment of industrial alliances of IOV, unify service interface standards, and form a situation where the government is the guide and enterprises participate in business competition. In the process of promoting the development of IOV technology, the following aspects should also be paid attention to:

Firstly, due to the long industrial chain of IOV and the large span of industries involved, it is necessary to integrate the complete industrial chain. Only by taking the lead of multiple administrative departments such as the ministry of communications, ministry of public security, ministry of construction and ministry of industry and information technology, can communication and coordination ability be guaranteed and multi-win be realized.

Second, there is a need for uniform standards of connectivity so that internet-connected vehicles, applications and services can be seamlessly integrated across all vertical markets. Only by forming and promulgating a complete and unified standard system and opening the system interface and application platform can we break down the barriers between industries and avoid separate governance. The key technical elements involved in the standardized solution, including network infrastructure, equipment, application, platform, security strategy and integrated services, are the severe challenges facing the implementation of the IOV planning and development.

Third, we still need to be integrated application of a variety of communication technology, Internet technology and cloud computing technology, to create the end-to-end vehicle networking information exchange and communication platform for the support and business to provide comprehensive, enrich the various types of business applications, actively explore and form can be well versed in upstream and downstream industries, safe, efficient, interactive smart vehicle networking business operation mode.

\section{References}

[1] Mohamed Nahri, Azedine Boulmakoul, Lamia Karim and Ahmed Lbath. IoV distributed architecture for real-time traffic data analytics [J]. Procedia Computer Science, Volume 130, p480-487, 2018.
[2] Danda B. Rawat, Reham Alsabet, Chandra Bajracharya and Min Song. On the performance of cognitive internet-of-vehicles with unlicensed user-mobility and licensed user-activity. Computer Networks, p98-106, 2018.

[3] W. Y. Lei, G. C. Wu, X. X. Tao, L. Bian and X. L. Wang. BDS satellite-induced code multipath: Mitigation and assessment in new-generation IOV satellites. Advances in Space Research, v60, n12, p2672-2679, December 2017.

[4] Ali Safa Sadiq, Suleman Khan, Kayhan Zrar Ghafoor, Mohsen Guizani and Seyedali Mirjalili. Transmission power adaption scheme for improving IoV awareness exploiting: evaluation weighted matrix based on piggybacked information. Computer Networks, v137, p147-159, June 2018.

[5] Tao Lei, Shangguang Wang, Jinglin Li and Fangchun Yang. A cooperative route choice approach via virtual vehicle in IoV. Vehicular Communications, v9, p281-287, July 2017.

[6] Han-Tao Zhao, Xin-Ru Liu, Xiao-Xu Chen and Jian-Cheng Lu. Cellular automata model for traffic flow at intersections in internet of vehicles. Physica A: Statistical Mechanics and its Applications, v494, p40-51, March 2018.

[7] Neeraj Kumar, Kuljeet Kaur, Anish Jindal and Joel J. P. C. Rodrigues. Providing healthcare services on-the-fly using multiplayer cooperation game theory in Internet of Vehicles (IoV) environment. Digital Communications and Networks, v1, n3, p191-203, August 2015.

[8] Fanhui Kong, Jian Li, Bin Jiang and Houbing Song. Short-term traffic flow prediction in smart multimedia system for Internet of Vehicles based on deep belief network. Future Generation Computer Systems, v93, p460-472, April 2019.

[9] Qinglei Kong, Rongxing Lu, Maode Ma and Haiyong Bao. A privacy-preserving sensory data sharing scheme in Internet of Vehicles. Future Generation Computer Systems, v92, p644-655, March 2019.

[10] Ming Tao, Wenhong Wei and Shuqiang Huang. Location-based trustworthy services recommendation in cooperativecommunication-enabled Internet of Vehicles. Journal of Network and Computer Applications, v126, p1-11, January 2019.

[11] Zhao Ting, Wang Bin and Gao Qi. Congestion warning method based on the Internet of vehicles and community discovery of complex networks. The Journal of China Universities of Posts and Telecommunications, v23, n4, p37-45, August 2016.

[12] Chen Chen, Xiaomin Liu, Tie Qiu, Lei Liu and Arun Kumar Sangaiah. Latency estimation based on traffic density for video streaming in the internet of vehicles. Computer Communications, v111, p176-186, October 2017.

[13] Min Chen, Yuanwen Tian, Giancarlo Fortino, Jing Zhang and Iztok Humar. Cognitive Internet of Vehicles. Computer Communications, v120, p58-70, May 2018.

[14] Bindiya Jain, Gursewak Brar, Jyoteesh Malhotra, Shalli Rani and Syed Hassan Ahmed. A cross layer protocol for traffic management in Social Internet of Vehicles. Future Generation Computer Systems, v82, p707-714, May 2018.

[15] Chen Chen, Hongyu Xiang, Tie Qiu, and etc. A rear-end collision prediction scheme based on deep learning in the Internet of Vehicles. Journal of Parallel and Distributed Computing, v117, p192-204, July 2018. 